Acta Crystallographica Section E

Structure Reports

Online

ISSN 1600-5368

\section{R. Allan}

School of Chemistry, The University of Edinburgh, King's Buildings, West Mains Road, Edinburgh EH9 3JJ, Scotland

Correspondence e-mail: d.r.allan@ed.ac.uk

\section{Key indicators}

Single-crystal synchrotron study

$T=205 \mathrm{~K}$

Mean $\sigma(\mathrm{C}-\mathrm{C})=0.003 \AA$

$R$ factor $=0.063$

$w R$ factor $=0.072$

Data-to-parameter ratio $=12.0$

For details of how these key indicators were automatically derived from the article, see http://journals.iucr.org/e.

\title{
Cyclobutylamine hemihydrate
}

The asymmetric unit of cyclobutylamine hemihydrate, $\mathrm{C}_{4} \mathrm{H}_{9} \mathrm{~N} \cdot 0.5 \mathrm{H}_{2} \mathrm{O}$, consists of two cyclobutylamine molecules bridged by a water molecule via $\mathrm{N} \cdots \mathrm{H}-\mathrm{O}$ hydrogen bonds. This molecular arrangement is further connected by significantly weaker $\mathrm{N}-\mathrm{H} \cdots \mathrm{O}$ contacts to form columns parallel to the $b$ axis.

\section{Comment}

The crystal structure of cyclobutylamine hemihydrate $\left(\mathrm{C}_{4} \mathrm{H}_{7} \mathrm{NH}_{2} \cdot 0.5 \mathrm{H}_{2} \mathrm{O}\right)$, (I), was determined at $205 \mathrm{~K}$ (just below the $\sim 210 \mathrm{~K}$ melting point) as part of our low-temperature and high-pressure structural studies of prototypical hydrogenbonded molecular systems. It crystallizes in the monoclinic space group $P 2_{1} / n$ with two cyclobutylamine molecules and one water molecule in the asymmetric unit (Fig. 1). Pairs of cyclobutylamine molecules are bridged by a single water molecule through $\mathrm{N} \cdots \mathrm{H}-\mathrm{O}$ hydrogen bonds, which have $\mathrm{N}$...O distances of $2.880(3)$ and 2.895 (2) $\AA$ (Fig. 2 and Table 1). Significantly weaker $\mathrm{N}-\mathrm{H} \cdots \mathrm{O}$ contacts link this molecular assembly to form columns parallel to the $b$ axis, with $\mathrm{N}$... O distances ranging in length from $3.176(3)$ and 3.281 (3) $\AA$ to a more marginal distance of 3.604 (3) $\AA$. As the $\mathrm{N}$... O distances increase, there is a concomitant decrease in the $\mathrm{N}-\mathrm{H} \cdots \mathrm{O}$ angles from $173.0(19)$ to $160.1(19)^{\circ}$ as the interaction weakens. The remaining $\mathrm{N}-\mathrm{H} \cdots \mathrm{O}$ interaction (N11-H111...O 1) would appear to link the columns into slabs parallel to (101). However, as this interaction has a very long $\mathrm{N} \cdots \mathrm{O}$ contact distance of 3.833 (3) $\AA$, and the $\mathrm{N}-\mathrm{H} \cdots \mathrm{O}$ angle is $134.3(15)^{\circ}$, it is unlikely to offer any significant contribution to the intermolecular bonding.

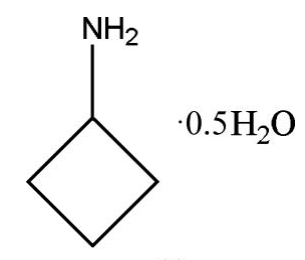

(I)
Received 10 January 2006 Accepted 18 January 2006 Online 25 January 2006
(C) 2006 International Union of Crystallography Printed in Great Britain - all rights reserved

\section{Experimental}

The sample of cyclobutylamine hemihydrate was prepared from anhydrous starting material (of $99 \%$ purity, as received from Aldrich) and placed in a sealed glass capillary tube with an internal diameter of ca $0.2 \mathrm{~mm}$. The sample was cooled using an Oxford Cryosystems lowtemperature device (Cosier \& Glazer, 1986) until crystallization was observed. The temperature was then cycled, by successive translations of the capillary through the gas stream, so that the sample was 
partially remelted and the number of crystallites reduced, until a single crystal was obtained at $205 \mathrm{~K}$.

\section{Crystal data \\ $\mathrm{C}_{4} \mathrm{H}_{9} \mathrm{~N} \cdot 0.5 \mathrm{H}_{2} \mathrm{O}$ \\ $M_{r}=80.13$ \\ Monoclinic, $P 2_{1} / n$ \\ $a=14.048(6) \AA$ \\ $b=5.209(2) \AA$ \\ $c=14.489(6) \AA$ \\ $\beta=97.369(4)^{\circ}$ \\ $V=1051.5(7) \AA^{3}$ \\ $Z=8$ \\ $D_{x}=1.012 \mathrm{Mg} \mathrm{m}^{-3}$}

\section{Data collection}

Bruker SMART diffractometer

$\omega$ scans

Absorption correction: multi-scan

(SADABS; Sheldrick, 2004)

$T_{\min }=0.55, T_{\max }=0.99$

8565 measured reflections

2525 independent reflections

\section{Refinement}

Refinement on $F$

$R\left[F^{2}>2 \sigma\left(F^{2}\right)\right]=0.063$

$w R\left(F^{2}\right)=0.072$

$S=1.14$

1411 reflections

118 parameters

$\mathrm{H}$ atoms treated by a mixture of independent and constrained refinement

Table 1

Hydrogen-bond geometry $\left(\AA,^{\circ}\right)$.

\begin{tabular}{lllll}
\hline$D-\mathrm{H} \cdots A$ & $D-\mathrm{H}$ & $\mathrm{H} \cdots A$ & $D \cdots A$ & $D-\mathrm{H} \cdots A$ \\
\hline $\mathrm{O} 1-\mathrm{H} 1 \cdots \mathrm{N} 11$ & $0.82(1)$ & $2.08(1)$ & $2.895(2)$ & $174(3)$ \\
$\mathrm{O} 1-\mathrm{H} 2 \cdots \mathrm{N} 21$ & $0.82(1)$ & $2.07(1)$ & $2.880(3)$ & $174(3)$ \\
\hline
\end{tabular}

$\mathrm{H}$ atoms attached to $\mathrm{C}$ atoms were placed in idealized positions $(\mathrm{C}-\mathrm{H}=0.94-1.00 \AA)$ and allowed to ride on their parent atoms. $\mathrm{H}$ atoms attached to $\mathrm{N}$ and $\mathrm{O}$ atoms were located in a difference map and restrained to idealized distances and angles $[\mathrm{N}-\mathrm{H}=0.90$ (1) $\AA$, $\mathrm{O}-\mathrm{H}=0.82(1) \AA$ and $\left.\mathrm{O}-\mathrm{H}-\mathrm{O}=104(1)^{\circ}\right]$. All $\mathrm{H}$ atoms were constrained so that $U_{\text {iso }}(\mathrm{H})$ was equal to $1.2 U_{\text {eq }}$ of their respective parent atoms.

Data collection: SMART (Bruker, 2001); cell refinement: SAINT; data reduction: SAINT (Bruker, 2003); program(s) used to solve structure: SIR92 (Altomare et al., 1994); program(s) used to refine structure: CRYSTALS (Betteridge et al., 2003); molecular graphics: CAMERON (Watkin et al., 1996); software used to prepare material for publication: CRYSTALS and PLATON (Spek, 2003).

We thank Dr T. Prior of Daresbury Laboratory for his help during the experiment on station 9.8 at SRS. We also thank the EPSRC for funding both this project and DRA's Advanced Research Fellowship.

\section{References}

Altomare, A., Burla, M. C., Camalli, G., Cascarano, G., Giacovazzo, C., Guagliardi, A. \& Polidori, G. (1994). J. Appl. Cryst. 27, 435-436.

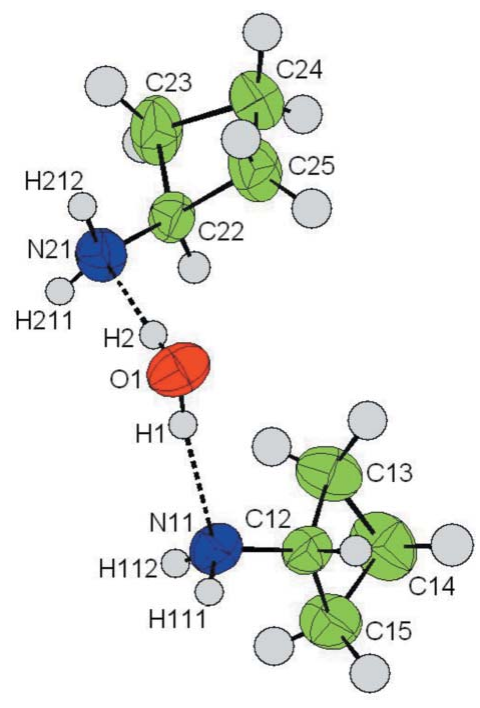

Figure 1

The asymmetric unit of (I), showing $30 \%$ probability displacement ellipsoids. The dashed lines indicate the $\mathrm{O}-\mathrm{H} \cdots \mathrm{N}$ hydrogen bonds.

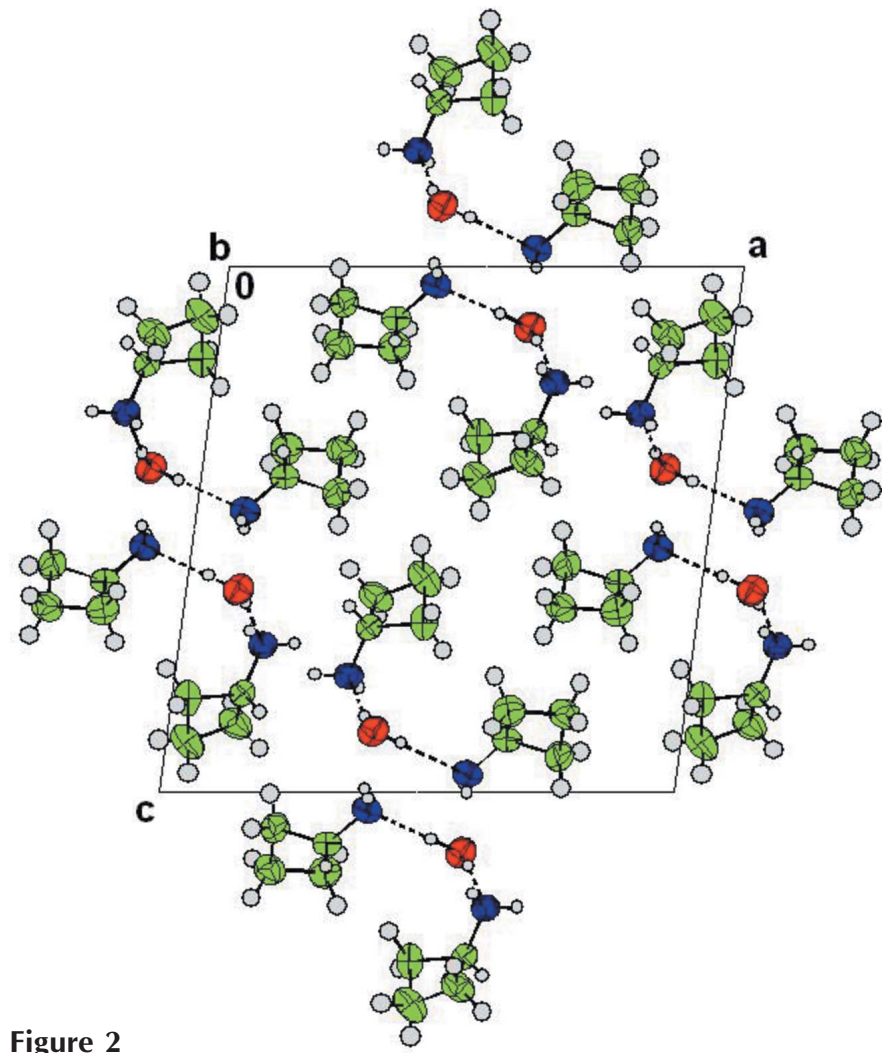

The packing of (I), viewed along the $b$ axis. The $\mathrm{O}-\mathrm{H} \cdots \mathrm{N}$ hydrogen bonds are shown as dashed lines.

Betteridge, P. W., Carruthers, J. R., Cooper, R. I., Prout, K. \& Watkin, D. J. (2003). J. Appl. Cryst. 36, 1487.

Bruker (2001). SMART. Bruker AXS Inc., Madison, Wisconsin, USA.

Bruker (2003). SAINT. Bruker AXS Inc., Madison, Wisconsin, USA.

Cosier, J. \& Glazer, A. M. (1986). J. Appl. Cryst. 19, 105-107.

Prince, E. (1982). Mathematical Techniques in Crystallography and Materials Science. New York: Springer-Verlag.

Sheldrick, G. M. (2004). SADABS. University of Gottingen, Germany.

Spek, A. L. (2003). J. Appl. Cryst. 36, 7-13.

Watkin, D. J. (1994). Acta Cryst. A50, 411-437.

Watkin, D. J., Prout, C. K. \& Pearce, L. (1996). CAMERON. Chemical Crystallography Laboratory, University of Oxford, England. 


\title{
supporting information
}

Acta Cryst. (2006). E62, o751-o752 [https://doi.org/10.1107/S1600536806002327]

\section{Cyclobutylamine hemihydrate}

\author{
D. R. Allan
}

cyclobutylamine hemihydrate

Crystal data

$\mathrm{C}_{4} \mathrm{H}_{9} \mathrm{~N} \cdot 0.5 \mathrm{H}_{2} \mathrm{O}$

$F(000)=360$

$M_{r}=80.13$

Monoclinic, $P 2_{1} / n$

Hall symbol: -P 2 yn

$a=14.048(6) \AA$

$b=5.209(2) \AA$

$c=14.489(6) \AA$

$\beta=97.369(4)^{\circ}$

$V=1051.5(7) \AA^{3}$

$Z=8$

$D_{\mathrm{x}}=1.012 \mathrm{Mg} \mathrm{m}^{-3}$

Synchrotron radiation, $\lambda=0.68130 \AA$

Cell parameters from 2051 reflections

$\theta=8-46^{\circ}$

$\mu=0.07 \mathrm{~mm}^{-1}$

$T=205 \mathrm{~K}$

Cylinder, colourless

$0.20 \times 0.20 \times 0.20 \times 0.20$ (radius) $\mathrm{mm}$

\section{Data collection}

Bruker SMART

diffractometer

Curved silicon monochromator

$\omega / 2 \theta$ scans

Absorption correction: multi-scan

(SADABS; Sheldrick, 2004)

$T_{\text {min }}=0.55, T_{\max }=0.99$

8565 measured reflections

2525 independent reflections

1411 reflections with $I>2 \sigma(I)$

$R_{\text {int }}=0.071$

$\theta_{\max }=27.5^{\circ}, \theta_{\min }=4.0^{\circ}$

$h=-18 \rightarrow 19$

$k=-6 \rightarrow 6$

$l=-19 \rightarrow 18$

\section{Refinement}

Refinement on $F$

Least-squares matrix: full

$R\left[F^{2}>2 \sigma\left(F^{2}\right)\right]=0.063$

$w R\left(F^{2}\right)=0.072$

$S=1.14$

1411 reflections

118 parameters

7 restraints

Primary atom site location: structure-invariant direct methods

Hydrogen site location: inferred from neighbouring sites

$\mathrm{H}$ atoms treated by a mixture of independent and constrained refinement

$w=\left[1-\left(F_{\mathrm{o}}-F_{\mathrm{c}}\right)^{2} / 36 \sigma^{2}(F)\right]^{2} /\left[2.28 \mathrm{~T}_{\mathrm{o}}(\mathrm{x})+\right.$ $\left.0.243 \mathrm{~T}_{1}(\mathrm{x})+1.74 \mathrm{~T}_{2}(\mathrm{x})\right]$

where $\mathrm{T}_{\mathrm{i}}$ are the Chebychev polynomials and $\mathrm{x}$

$=F_{\mathrm{d}} / F_{\max }($ Prince, 1982; Watkin, 1994)

$(\Delta / \sigma)_{\max }=0.000218$

$\Delta \rho_{\max }=0.17 \mathrm{e} \AA^{-3}$

$\Delta \rho_{\min }=-0.18$ e $\AA^{-3}$ 


\section{Special details}

Refinement. ABSTM02_ALERT_3_B The ratio of expected to reported Tmax/Tmin $\left(R R^{\prime}\right)$ is $<0.75 \mathrm{~T} \min$ and Tmax reported: $0.5500 .990 \mathrm{~T} \min$ (prime) and Tmax expected: $0.9870 .987 R R$ (prime) $=0.556$

$S A D A B S$ was also used to correct for the decay of the synchrotron X-ray beam. The overall sample absorption, especially at the relatively short wavelength, is extremely low.

PLAT241_ALERT_2_C Check High $U_{\text {eq }}$ as Compared to Neighbors for C23 PLAT242_ALERT_2_C Check Low $U_{\text {eq }}$ as Compared to Neighbors for C12 PLAT242_ALERT_2_C Check Low $U_{\text {eq }}$ as Compared to Neighbors for C22

The data were collected very close to the sample melting temperature and, consequently, the temperature factors are relatively large.

PLAT420_ALERT_2_C D-H Without Acceptor N11 - H111 … ? PLAT420_ALERT_2_C D-H Without Acceptor N21 - $\mathrm{H} 211 \cdots \bar{?}$ ?

Although the relevant $\mathrm{N}-\mathrm{H} \cdots \mathrm{O}$ angles suggest that the oxygen atom acts as an acceptor for both $\mathrm{N} 11-\mathrm{H} 111$ and $\mathrm{N} 21-$ $\mathrm{H} 211$, the $\mathrm{H} \cdots \mathrm{A}$ distances are relatively long and suggest that these interactions are at best extremely weak. Details of the various distances are mentioned in the comments section.

Fractional atomic coordinates and isotropic or equivalent isotropic displacement parameters $\left(\AA^{2}\right)$

\begin{tabular}{|c|c|c|c|c|}
\hline & $x$ & $y$ & $z$ & $U_{\text {iso }} * / U_{\text {eq }}$ \\
\hline N11 & $0.66281(12)$ & 0.6261 & $0.22065(10)$ & 0.0590 \\
\hline $\mathrm{C} 12$ & $0.63480(12)$ & 0.6188 & $0.31320(12)$ & 0.0524 \\
\hline C13 & $0.52996(14)$ & $0.5731(4)$ & $0.32271(17)$ & 0.0742 \\
\hline $\mathrm{C} 14$ & $0.54031(19)$ & $0.7577(5)$ & $0.40528(19)$ & 0.0882 \\
\hline $\mathrm{C} 15$ & $0.62917(17)$ & $0.8626(4)$ & $0.37068(15)$ & 0.0751 \\
\hline $\mathrm{O} 1$ & $0.60087(11)$ & $0.1702(3)$ & $0.11547(10)$ & 0.0705 \\
\hline N21 & $0.40677(12)$ & $0.2763(3)$ & $0.03446(11)$ & 0.0623 \\
\hline $\mathrm{C} 22$ & $0.33839(13)$ & $0.2419(4)$ & $0.09937(11)$ & 0.0534 \\
\hline $\mathrm{C} 25$ & $0.34180(17)$ & $-0.0039(5)$ & $0.15398(16)$ & 0.0773 \\
\hline $\mathrm{C} 24$ & $0.23267(16)$ & $0.0020(5)$ & $0.14836(15)$ & 0.0752 \\
\hline $\mathrm{C} 23$ & $0.23237(15)$ & 0.1925 (6) & $0.06886(15)$ & 0.0846 \\
\hline H121 & 0.6732 & 0.4839 & 0.3504 & $0.0625 *$ \\
\hline H131 & 0.5138 & 0.3963 & 0.3372 & $0.0889 *$ \\
\hline H132 & 0.4884 & 0.6336 & 0.2675 & $0.0894 *$ \\
\hline H141 & 0.5514 & 0.6690 & 0.4633 & $0.1089 *$ \\
\hline H142 & 0.4882 & 0.8755 & 0.4054 & $0.1088^{*}$ \\
\hline H151 & 0.6820 & 0.8860 & 0.4183 & $0.0917^{*}$ \\
\hline H152 & 0.6191 & 1.0139 & 0.3349 & $0.0923 *$ \\
\hline $\mathrm{H} 221$ & 0.3435 & 0.3886 & 0.1421 & $0.0646^{*}$ \\
\hline $\mathrm{H} 251$ & 0.3778 & 0.0052 & 0.2178 & $0.0935^{*}$ \\
\hline $\mathrm{H} 252$ & 0.3650 & -0.1418 & 0.1190 & $0.0938^{*}$ \\
\hline H241 & 0.2105 & 0.0777 & 0.2026 & $0.0910^{*}$ \\
\hline $\mathrm{H} 242$ & 0.1995 & -0.1578 & 0.1341 & $0.0915^{*}$ \\
\hline H231 & 0.1894 & 0.3458 & 0.0706 & $0.1014^{*}$ \\
\hline H232 & 0.2216 & 0.1046 & 0.0084 & $0.1013^{*}$ \\
\hline H211 & $0.4070(17)$ & $0.435(2)$ & $0.0116(15)$ & $0.0744^{*}$ \\
\hline H1 & $0.6198(15)$ & $0.303(3)$ & $0.1417(16)$ & $0.1011^{*}$ \\
\hline $\mathrm{H} 2$ & $0.5447(9)$ & $0.202(5)$ & $0.0966(18)$ & $0.1017^{*}$ \\
\hline $\mathrm{H} 212$ & $0.4029(16)$ & $0.142(3)$ & $-0.0039(13)$ & $0.0737^{*}$ \\
\hline H111 & $0.7268(7)$ & $0.634(4)$ & $0.2227(14)$ & $0.0715^{*}$ \\
\hline H112 & $0.6347(14)$ & $0.767(3)$ & $0.1950(14)$ & $0.0719^{*}$ \\
\hline
\end{tabular}


Atomic displacement parameters $\left(\AA^{2}\right)$

\begin{tabular}{lllllll}
\hline & $U^{11}$ & $U^{22}$ & $U^{33}$ & $U^{12}$ & $U^{13}$ & $U^{23}$ \\
\hline $\mathrm{N} 11$ & $0.0650(9)$ & $0.0589(9)$ & $0.0525(8)$ & $-0.0002(7)$ & $0.0051(7)$ & $-0.0081(7)$ \\
$\mathrm{C} 12$ & $0.0524(9)$ & $0.0517(9)$ & $0.0517(9)$ & $0.0010(7)$ & $0.0018(7)$ & $-0.0005(7)$ \\
$\mathrm{C} 13$ & $0.0556(11)$ & $0.0703(13)$ & $0.0975(15)$ & $-0.0014(9)$ & $0.0131(10)$ & $-0.0004(12)$ \\
$\mathrm{C} 14$ & $0.0908(16)$ & $0.0859(16)$ & $0.0964(16)$ & $0.0068(13)$ & $0.0448(13)$ & $-0.0057(14)$ \\
$\mathrm{C} 15$ & $0.0918(15)$ & $0.0652(12)$ & $0.0728(13)$ & $-0.0111(10)$ & $0.0278(11)$ & $-0.0215(10)$ \\
O1 & $0.0712(9)$ & $0.0643(9)$ & $0.0741(9)$ & $0.0055(7)$ & $0.0025(7)$ & $-0.0171(7)$ \\
N21 & $0.0700(10)$ & $0.0620(10)$ & $0.0558(8)$ & $-0.0048(8)$ & $0.0120(7)$ & $0.0000(8)$ \\
C22 & $0.0667(10)$ & $0.0482(9)$ & $0.0447(8)$ & $0.0021(8)$ & $0.0054(7)$ & $-0.0016(7)$ \\
C25 & $0.0811(14)$ & $0.0738(14)$ & $0.0764(13)$ & $0.0088(11)$ & $0.0073(10)$ & $0.0255(11)$ \\
C24 & $0.0802(14)$ & $0.0782(15)$ & $0.0694(13)$ & $-0.0122(11)$ & $0.0181(10)$ & $0.0092(11)$ \\
C23 & $0.0596(11)$ & $0.122(2)$ & $0.0709(12)$ & $0.0029(12)$ & $0.0025(9)$ & $0.0293(13)$ \\
& & & & & &
\end{tabular}

Geometric parameters $\left(A,{ }^{\circ}\right)$

\begin{tabular}{|c|c|c|c|}
\hline $\mathrm{N} 11-\mathrm{C} 12$ & $1.446(2)$ & $\mathrm{O} 1-\mathrm{H} 2$ & $0.819(10)$ \\
\hline N11-H111 & $0.896(9)$ & $\mathrm{N} 21-\mathrm{C} 22$ & $1.439(2)$ \\
\hline N11-H112 & $0.892(9)$ & $\mathrm{N} 21-\mathrm{H} 211$ & $0.892(9)$ \\
\hline $\mathrm{C} 12-\mathrm{C} 13$ & $1.516(3)$ & $\mathrm{N} 21-\mathrm{H} 212$ & $0.892(9)$ \\
\hline $\mathrm{C} 12-\mathrm{C} 15$ & $1.526(3)$ & $\mathrm{C} 22-\mathrm{C} 25$ & $1.502(3)$ \\
\hline $\mathrm{C} 12-\mathrm{H} 121$ & 1.001 & $\mathrm{C} 22-\mathrm{C} 23$ & $1.520(3)$ \\
\hline $\mathrm{C} 13-\mathrm{C} 14$ & $1.527(4)$ & $\mathrm{C} 22-\mathrm{H} 221$ & 0.980 \\
\hline $\mathrm{C} 13-\mathrm{H} 131$ & 0.977 & $\mathrm{C} 25-\mathrm{C} 24$ & $1.525(3)$ \\
\hline $\mathrm{C} 13-\mathrm{H} 132$ & 0.980 & $\mathrm{C} 25-\mathrm{H} 251$ & 0.996 \\
\hline $\mathrm{C} 14-\mathrm{C} 15$ & $1.506(3)$ & $\mathrm{C} 25-\mathrm{H} 252$ & 0.960 \\
\hline $\mathrm{C} 14-\mathrm{H} 141$ & 0.955 & $\mathrm{C} 24-\mathrm{C} 23$ & $1.520(3)$ \\
\hline $\mathrm{C} 14-\mathrm{H} 142$ & 0.955 & $\mathrm{C} 24-\mathrm{H} 241$ & 0.966 \\
\hline $\mathrm{C} 15-\mathrm{H} 151$ & 0.954 & $\mathrm{C} 24-\mathrm{H} 242$ & 0.963 \\
\hline $\mathrm{C} 15-\mathrm{H} 152$ & 0.944 & $\mathrm{C} 23-\mathrm{H} 231$ & 1.004 \\
\hline $\mathrm{O} 1-\mathrm{H} 1$ & $0.817(10)$ & $\mathrm{C} 23-\mathrm{H} 232$ & 0.983 \\
\hline $\mathrm{C} 12-\mathrm{N} 11-\mathrm{H} 111$ & $111.2(14)$ & $\mathrm{C} 22-\mathrm{N} 21-\mathrm{H} 211$ & $113.2(15)$ \\
\hline $\mathrm{C} 12-\mathrm{N} 11-\mathrm{H} 112$ & $104.5(14)$ & $\mathrm{C} 22-\mathrm{N} 21-\mathrm{H} 212$ & $108.5(14)$ \\
\hline H111-N11-H112 & $111.5(19)$ & $\mathrm{H} 211-\mathrm{N} 21-\mathrm{H} 212$ & $120(2)$ \\
\hline $\mathrm{N} 11-\mathrm{C} 12-\mathrm{C} 13$ & $118.15(16)$ & $\mathrm{N} 21-\mathrm{C} 22-\mathrm{C} 25$ & $118.13(17)$ \\
\hline $\mathrm{N} 11-\mathrm{C} 12-\mathrm{C} 15$ & $121.58(16)$ & $\mathrm{N} 21-\mathrm{C} 22-\mathrm{C} 23$ & $122.83(15)$ \\
\hline $\mathrm{C} 13-\mathrm{C} 12-\mathrm{C} 15$ & $87.84(15)$ & $\mathrm{C} 25-\mathrm{C} 22-\mathrm{C} 23$ & $88.44(17)$ \\
\hline $\mathrm{N} 11-\mathrm{C} 12-\mathrm{H} 121$ & 109.1 & $\mathrm{~N} 21-\mathrm{C} 22-\mathrm{H} 221$ & 108.3 \\
\hline $\mathrm{C} 13-\mathrm{C} 12-\mathrm{H} 121$ & 107.7 & $\mathrm{C} 25-\mathrm{C} 22-\mathrm{H} 221$ & 109.7 \\
\hline $\mathrm{C} 15-\mathrm{C} 12-\mathrm{H} 121$ & 110.6 & $\mathrm{C} 23-\mathrm{C} 22-\mathrm{H} 221$ & 107.8 \\
\hline $\mathrm{C} 12-\mathrm{C} 13-\mathrm{C} 14$ & $88.64(17)$ & $\mathrm{C} 22-\mathrm{C} 25-\mathrm{C} 24$ & $89.49(16)$ \\
\hline $\mathrm{C} 12-\mathrm{C} 13-\mathrm{H} 131$ & 115.0 & $\mathrm{C} 22-\mathrm{C} 25-\mathrm{H} 251$ & 115.2 \\
\hline $\mathrm{C} 14-\mathrm{C} 13-\mathrm{H} 131$ & 115.2 & $\mathrm{C} 24-\mathrm{C} 25-\mathrm{H} 251$ & 115.9 \\
\hline $\mathrm{C} 12-\mathrm{C} 13-\mathrm{H} 132$ & 111.1 & $\mathrm{C} 22-\mathrm{C} 25-\mathrm{H} 252$ & 110.5 \\
\hline $\mathrm{C} 14-\mathrm{C} 13-\mathrm{H} 132$ & 115.1 & $\mathrm{C} 24-\mathrm{C} 25-\mathrm{H} 252$ & 113.2 \\
\hline $\mathrm{H} 131-\mathrm{C} 13-\mathrm{H} 132$ & 110.4 & $\mathrm{H} 251-\mathrm{C} 25-\mathrm{H} 252$ & 111.0 \\
\hline
\end{tabular}


$\mathrm{C} 13-\mathrm{C} 14-\mathrm{C} 15$

C13-C14-H141

$\mathrm{C} 15-\mathrm{C} 14-\mathrm{H} 141$

C13-C14-H142

C15-C14-H142

H141-C14-H142

$\mathrm{C} 12-\mathrm{C} 15-\mathrm{C} 14$

C12-C15-H151

C14-C15-H151

C12-C15-H152

C14-C15-H152

H151-C15-H152

$\mathrm{H} 1-\mathrm{O} 1-\mathrm{H} 2$
88.14 (16)

111.9

114.9

114.2

115.8

110.3

89.01 (17)

114.3

114.0

114.2

114.5

109.8

103 (2)
$\mathrm{C} 25-\mathrm{C} 24-\mathrm{C} 23$

$\mathrm{C} 25-\mathrm{C} 24-\mathrm{H} 241$

$\mathrm{C} 23-\mathrm{C} 24-\mathrm{H} 241$

$\mathrm{C} 25-\mathrm{C} 24-\mathrm{H} 242$

$\mathrm{C} 23-\mathrm{C} 24-\mathrm{H} 242$

$\mathrm{H} 241-\mathrm{C} 24-\mathrm{H} 242$

$\mathrm{C} 22-\mathrm{C} 23-\mathrm{C} 24$

$\mathrm{C} 22-\mathrm{C} 23-\mathrm{H} 231$

$\mathrm{C} 24-\mathrm{C} 23-\mathrm{H} 231$

$\mathrm{C} 22-\mathrm{C} 23-\mathrm{H} 232$

$\mathrm{C} 24-\mathrm{C} 23-\mathrm{H} 232$

$\mathrm{H} 231-\mathrm{C} 23-\mathrm{H} 232$
$87.61(15)$

113.1

112.4

116.7

116.6

109.2

89.02 (15)

115.4

116.3

111.8

110.9

111.6

Hydrogen-bond geometry $\left(A,{ }^{\circ}\right)$

\begin{tabular}{lllll}
\hline$D-\mathrm{H} \cdots A$ & $D-\mathrm{H}$ & $\mathrm{H} \cdots A$ & $D \cdots A$ & $D-\mathrm{H} \cdots A$ \\
\hline $\mathrm{O} 1-\mathrm{H} 1 \cdots \mathrm{N} 11$ & $0.82(1)$ & $2.08(1)$ & $2.895(2)$ & $174(3)$ \\
$\mathrm{O} 1-\mathrm{H} 2 \cdots \mathrm{N} 21$ & $0.82(1)$ & $2.07(1)$ & $2.880(3)$ & $174(3)$ \\
\hline
\end{tabular}

